\section{Tiempo de cirugía efectiva en la extracción de los terceros molares realizadas por un cirujano oral y maxilofacial con experiencia}

Llerena G, Arrascue M. Tiempo de cirugía efectiva en la extracción de los terceros molares realizadas por un cirujano oral y maxilofacial con experiencia. Rev Estomatol Herediana 2006; 16(1): $40-45$.

\section{RESUMEN}

Se examinaron radiográficamente 100 terceros molares inferiores indicados para extraer y se tomó registro de las historias clínicas respectivas, para obtener las clasificaciones de PellGregory, Winter y las formas de las raíces comparando estas variables con el tiempo de cirugía efectiva al realizar las extracciones por un Cirujano Oral y Maxilofacial con experiencia. El tiempo de cirugía efectiva promedio de la exodoncia del tercer molar inferior fue de 12,71 min. Se encontró con mayor frecuencia la posición mesioangular, el nivel A y la clase II. La forma más frecuente de las raíces del tercer molar inferior fue completa múltiple y dilacerada El motivo más frecuente para realizar la exodoncia fue el preventivo. Dentro de los factores que determinan la ubicación del diente, sólo la clase III guardaba relación directa con el tiempo de cirugía efectiva al realizar la exodoncia mientras que la posición vertical tuvo una relación inversamente proporcional. No hubo relación significativa entre el tiempo de cirugía efectiva y la forma de raíces, pero si una relación directamente proporcional y significativa con la edad del paciente.

Palabras clave: CIRUGÍA BUCAL / TERCER MOLAR.

Effective surgical time in third molar extractions performed by an experienced oral and maxillofacial surgeon.

ABSTRACT

One hundred inferior third molar teeth with extraction indication were evaluated through panoramic radiographs and clinical histories to obtain Pell-Gregory's, Winter's and root shape classifications. All variables were compared with the effective time of third molar surgical extractions performed by an experienced oral and maxillofacial surgeon. Effective surgical time average for all extracted teeth was $12,71 \mathrm{~min}$. Most frequently teeth position was mesioangular, level A, class II. The most common root shape was complete, multiple and dilacerated. The main reason for extraction was prevention. Only class III position had a direct relation with effective surgical time, while the vertical position had an inverse relation. There was no significant relation between time and root shape but a direct relation was found between time of surgery and age of the patient.

Keywords: ORAL SURGERY / THIRD MOLAR.
Giselle Llerena García ${ }^{1}$ Manuel Arrascue Dulanto ${ }^{2}$

${ }^{1}$ Cirujano Dentista

'Docente del Departamento Académico de Medicina, Cirugía y Patología Oral.

Facultad de Estomatología.

Universidad Peruana Cayetano Heredia.

\section{Correspondencia}

Giselle Llerena García

Calle Cosmos 451 Urb Las Gardenias - Lima 31, Perú

Teléfono 273274996438590

e-mail: gisellellerena@yahoo.com

Aceptado para publicación :

20 de noviembre del 2006

\section{Introducción}

Existen varias indicaciones para extraer un tercer molar inferior. El procedimiento de la extracción puede ser simple o complicado; para poder predecir el grado de complejidad es necesario conocer los factores que le afectan; así, antes de realizar el procedimiento quirúrgico es necesario hacer una evaluación detenida de los problemas que podrían presentarse y dificulten la extracción (1-3).

Las variantes anatómicas del tercer molar inferior, la disponibilidad de una radiografía, las condiciones en que se realizan los abordajes quirúrgicos, las posibles complicaciones intraoperatorias y la experiencia del operador pueden afectar el tiempo operatorio de una extracción de un tercer molar. Es por ello que el tercer molar inferior es un cons- tante problema para los cirujanos ya que su posición en relación con el diente adyacente y con la rama ascendente del hueso mandibular hacen difícil el acceso al mismo $(4,5)$.

El tercer molar inferior es el diente que presenta mayores variedades de forma, tamaño y ubicación. Generalmente, es birradicular, pero puede tener tres, cuatro y cinco raíces con una disposición más o menos caprichosa $(1,3)$. La anatomía de la raíz es importante, no sólo por su forma y dirección sino también por su relación con otras estructuras anatómicas. La variación en la anatomía radicular empeora el problema referido a la falta de espacio para extraer el tercer molar (1,6-8).

Es imprescindible realizar siempre un estudio radiológico preciso que muestre todo el tercer molar incluido y las estructuras que lo rodean $(1,7,9)$. Con una correcta interpretación radiográfica se pueden predecir los factores locales que influyen en la mayor o menor dificultad de la extracción. Entre los factores principales tenemos: el acceso al tercer molar, la profundidad y posición del mismo, la forma de la corona y raíces, cantidad de hueso que lo rodea y la relación con el nervio dentario inferior (1,8-11).

Teniendo en cuenta las consideraciones anatómicas y la posición de la pieza se obtendran mejores resultados post operatorios. Por este motivo se han clasificado diversos factores y creado índices que nos ayudan a predecir la dificultad de este acto quirúrgico (12).

Winter (1926) clasificó al tercer molar inferior según el ángulo que formaba su eje mayor en relación con el eje ma- 
yor del segundo molar (1). Pell y Gregory (1933) establecieron un índice basándose en la relación que existe entre el nivel del diente y el plano oclusal del segundo molar así como la relación entre el tercer molar y el espacio que existe entre la rama ascendente y el segundo molar (13,14). Archer (15) en 1979 presentó una clasificación exhaustiva de los terceros molares mandibulares relacionando el espacio existente, cuánto ha erupcionado y su relación longitudinal con la segunda molar.

Koerner (14) en 1995, muestra dos factores más dentro de su evaluación para medir la dificultad de la extracción, estos son edad y forma facial. Considera la edad como un indicador, cuya puntuación más elevada está asociada a los adultos mayores (más de 35 años) y una puntuación más baja a los más jóvenes, cuyas raíces no estaban totalmente formadas. Relaciona también la forma facial anatómica innata de cada persona con el acceso quirúrgico.

García et al. (13) en el 2000 evaluaron 166 extracciones del tercer molar inferior en posición vertical realizadas por un solo cirujano. Cada pieza fue clasificada según la escala de PellGregory como fácil o difícil. Sin embargo se encontró que las consideradas difíciles no lo fueron en el momento de la extracción.

Reyneke et al. (16) en 2002 observaron que la presencia de un tercer molar inferior no erupcionado aumentaba el grado de dificultad del acto quirúrgico durante la osteotomía.

En nuestro medio, Delgado (17) en 2001 realizó un estudio para determinar las diferentes características de un tercer molar observadas en las radiografías panorámicas, clasificando a las raíces en 16 tipos posibles, entre ellas raíces rectas, curvas separadas, fusionadas, con dirección distal o mesial, simples, múltiples y otras.

Revisando la literatura, se ha podido observar que aún cuando se habla mucho de las raíces, estas no son tomadas en cuenta al evaluar el tiempo de cirugía efectiva y cuanto aumenta el tiempo de la extracción a causa de esta variante. Muchas veces al extraer un tercer molar inferior impactado, lo primero que se retira es la corona, lo cual se realiza sin mayor dificultad. La dificultad se presenta al retirar la raíz del diente. Su variante anatomía la hace de difícil acceso e incluso puede sorprendernos en el mismo proceso quirúrgico. Por lo que debería incluirse dentro de los índices de dificultad la forma de las raíces para así poder conocer que tan complicado puede ser el procedimiento y el tiempo que tomará realizarlo (18).

El propósito de este estudio fue evaluar el tiempo de la cirugía efectiva de la exodoncia de terceros molares inferiores realizados por un especialista en cirugía oral y maxilofacial en su práctica privada, relacionando el tiempo con la ubicación y forma de las raíces

\section{Materiales y métodos}

Se realizaron 100 extracciones de terceros molares inferiores en la consulta privada de un especialista en Cirugía Oral y Maxilofacial con más de diez años de experiencia, durante un periódo de doce meses desde enero del 2003.

Estudio radiográfico. En todos los casos se tomó una radiografía periapical y en los casos donde existió alguna dificultad para la radiográfica intraoral, se tomó una radiografía panorámica.

Procedimientos pre-quirúrgicos. En todos los casos se contó con el consentimiento firmado por el paciente. Se utilizó una ficha de recolección de datos donde se registró la edad, sexo y ubicación de la pieza por hemiarcada y según las clasificaciones de Winter y Pell-Gregory. Se preparó al paciente con todas las normas de asepsia y antisepsia (1).

Anestesia. Se utilizó la técnica troncular para anestesiar la rama del dentario inferior y el nervio lingual, así como la técnica infiltrativa para el nervio bucal (1).

Acto quirúrgico. Al momento de iniciar el acto quirúrgico ya sea mediante una incisión o la realización de la sindesmotomía, se procedió a marcar con el cronómetro el tiempo de cirugía. Según los casos se realizó el colgajo adecuado para la correcta visualización de la pieza. Cuando no se requirió de incisión se procedió a realizar la sindesmotomía con los elevadores (1). A continuación se procedió a realizar la osteotomía hasta conseguir el espacio suficiente para la luxación de la pieza y su desplazamiento. Seguidamente se realizó la odontosección para conseguir la exéresis del tercer molar (1). Retirados todos los fragmentos y/o la pieza completa en sí, se realizó un curetaje, limpieza con agua estéril a presión y una buena succión para verificar que no permanezca alguna espícula de hueso o resto de diente (1).

Una vez realizada la extracción dentaria se procedió a detener el cronómetro. Considerando el momento final cuando se realizó la avulsión de la pieza. No se contabilizó el tiempo que tomó realizar la sutura, cuando fue necesario). Se dió las recomendaciones necesarias al paciente y se le indicó su cita de control a las 48 horas y a los cinco días para el retiro de puntos (1). Se registró el tiempo de la extracción en la ficha de recolección.

Plan de análisis. Se realizó un análisis univariado, calculando para el tiempo y la edad la desviación estándar y la media, así cómo sus valores mínimos y máximos, mientras que para ubicación, forma, sexo y hemiarcada se calculó las frecuencias absolutas y relativas. Se continúo con un análisis bivariado, en el cual se busco relacionar el tiempo con todas las variables a través del uso de coeficientes de correlación de Pearson y del análisis de regresión lineal simple. Finalmente se realizó un análisis multivariado para la confrontación de todas las variables, obteniendo en el modelo final sólo aquellas que presentaban una significancia menor a 0,25.

\section{Resultados}

Del total de 100 exodoncias de terceras molares inferiores evaluadas 63 fueron en mujeres y 52 estaban ubicadas en la arcada izquierda. El promedio de edad de los pacientes fue de 24 años, la edad minima fue de 15 años y la máxima 56 años y una desviación estándar de 6,79 .

El tiempo promedio de cirugía efectiva fue de 13,03 minutos con una des- 
viación estándar de 11,40 minutos, teniendo un tiempo mínimo de un minuto y un máximo de 55 minutos.

La ubicación de los terceros molares en cada caso se evalúo siguiendo los índices de Pell-Gregory y Winter, estudiando la relación espacial, la profundidad y el espacio disponible. La relación espacial fue: 55\% mesioangulares, 29\% verticales, 9\% distoangulares y 7\% horizontales. Respecto a la profundidad se obtuvo un orden de frecuencia de $70 \%$ en el nivel A, 25\% en el nivel B y 5\% en el nivel C. Y con respecto al espacio disponible se obtuvo un orden de frecuencia igual a 56\% para la clase II, 22\% para la clase I y III.

La forma de las raíces fue clasificada según su número y características radiográficas, obteniéndose las siguientes frecuencias: 54\% completa múltiple dilacerada, 22\% completa múltiple recta, 16\% incompletas, $8 \%$ completas únicas rectas. No se encontró ningún caso de completa única dilacerada.

La frecuencia con relación al motivo de la extracción fue en 37\% de los casos por motivos preventivos y 33\% por pericoronaritis. Sólo 18\% casos acudieron por motivos ortodónticos mientras que un total de $12 \%$ casos acudieron por diversos motivos cómo caries dental, absceso apical, enfermedad periodontal entre otros.

Una vez obtenidos estos datos se relacionó cada una de las variables con el factor tiempo a través de coeficientes de correlación y considerando la asociación significativa con un $\mathrm{p}<0,05$ utilizando la clasificación de Colton. Al relacionar el tiempo con la ubicación se encontró primero que con respecto a la relación espacial, el tiempo se veía afectado sólo cuando se presentaban las posiciones vertical, mesioangular y horizontal. Con respecto a la profundidad se observó que sólo eran significativas la presencia del Nivel A y B mientras que con relación al espacio disponible fueron significativas la presencia de la Clase I y III. (Tabla 1)

Cuando se relacionó el tiempo con la forma de las raíces no se observó que la presencia de algunas de las formas establecidas fuera significativa para la variación de éste (Tabla 2). Sin embargo, la edad fue una variable muy significativa cuando se relacionaba con el tiempo, encontrándose una relación directa entre ambos. Por tanto a mayor edad, mayor tiempo de cirugía efectiva (Tabla 3). Al relacionar el tiempo con el motivo por el cual se realizaban las exodoncias tampoco se observó una relación significativa entre ambos (Tabla 4).

Finalmente, al aplicar un análisis multivariado para correlacionar cada una de las variables estudiadas con el tiempo, se obtuvo que para poder predecir el tiempo sólo era necesario la presencia de la posición vertical, la clase III y la edad, cualquiera que ésta sea (Tabla 5). Se obtuvo entonces la siguiente fórmula: Tiempo=3,2-6,94 (Relación vertical)+7,04 (Clase III)+0,41 (Edad). Donde, tanto la presencia o ausencia de la relación vertical y la clase III toman los valores de 1 y 0 respectivamente según corresponda. Mientras que la edad tomará el valor correspondiente a la edad real de cada paciente.

Así al aplicar la fórmula, por ejemplo, en uno de los casos donde se observó un paciente masculino de 16 años de edad que presenta un tercer molar inferior derecho en posición mesioangular, nivel A, clase II, con las raíces completas múltiples y rectas se tiene que al reemplazar las variables se obtuvo la siguiente ecuación: Tiempo=3,2$(6,94 \times 0)+(7,04 \times 0)+(0,41 \times 16)=9,76$. El valor obtenido es el tiempo que se hubiera esperado encontrar. Al compararlo con el tiempo real obtenido que fue de 10 minutos, observamos que no había mucha diferencia.

Al aplicar la fórmula adquirida en todos los casos, se obtuvo un tiempo promedio igual a 12,71 minutos con una desviación estándar de 5,28 minutos. Presentando un tiempo mínimo de 2,41 minutos y un tiempo máximo igual a 26,76 minutos (Tabla 6).

Para la verificación de los datos obtenidos a través de sus medias, se aplicó la prueba t student para grupos relacionados, encontrándose que no existía diferencia significativa entre los tiempos obtenidos y esperados (Tabla 7).
Tabla 1. Relación entre el tiempo de cirugía efectiva de las exodoncias con respecto a la ubicación del diente.

\begin{tabular}{lcc}
\hline Ubicación & $\mathrm{r}$ & $\mathrm{p}$ \\
\hline Relación espacial & & \\
-Vertical & $-0,312$ & 0,001 \\
-Mesioangular & 0,181 & 0,035 \\
-Horizontal & 0,241 & 0,008 \\
-Distoangular & $-0,042$ & 0,339 \\
Profundidad & & \\
-Nivel A & $-0,189$ & 0,03 \\
-Nivel B & 0,235 & 0,009 \\
-Nivel C & $-0,069$ & 0,246 \\
Espacio disponible & & \\
-Clase I & $-0,278$ & 0,003 \\
-Clase II & $-0,012$ & 0,453 \\
-Clase III & 0,292 & 0,002 \\
\hline
\end{tabular}

Tabla 2. Relación entre el tiempo de cirugía efectiva de las exodoncias con respecto a la forma de las raíces.

\begin{tabular}{lcr}
\hline Forma & \multicolumn{1}{c}{$\mathrm{r}$} & \multicolumn{1}{c}{$\mathrm{p}$} \\
\hline Incompletas & $-0,018$ & 0,43 \\
C.U.R. & 0,015 & 0,439 \\
C.M.R. & 0,06 & 0,276 \\
C.U.D. & - & - \\
C.M.D. & $-0,045$ & 0,327 \\
\hline
\end{tabular}

Tabla 3. Relación entre el tiempo de cirugía efectiva de las exodoncias con respecto a la edad del paciente.

\begin{tabular}{lcc}
\hline Edad & $\mathrm{r}$ & $\mathrm{p}$ \\
\hline $\mathrm{X}$ & 0,233 & 0,01 \\
\hline
\end{tabular}

Tabla 4. Relación entre el tiempo de cirugía efectiva de las exodoncias con respecto al motivo de consulta.

\begin{tabular}{lcc}
\hline Motivo & $\mathrm{r}$ & $\mathrm{P}$ \\
\hline Preventivo & 0,044 & 0,333 \\
Ortodóncicos & $-0,062$ & 0,271 \\
Pericoronaritis & $-0,029$ & 0,388 \\
Otros & 0,044 & 0,333 \\
\hline
\end{tabular}


Tabla 5. Relación entre el tiempo de cirugía efectiva de las exodoncias con las variables mas significativas.

\begin{tabular}{lcccc}
\hline & $\mathrm{r}$ & $\mathrm{p}$ & Beta & Sig \\
\hline Vertical & $-0,312$ & 0,001 & $-6,945$ & 0,003 \\
Clase III & 0,292 & 0,002 & 7,41 & 0,004 \\
Edad & 0,233 & 0,01 & 0,416 & 0,007 \\
Cte. & & & 3,287 & 0,409 \\
\hline
\end{tabular}

Tabla 6. Tiempo de cirugía efectiva promedio esperado de las exodoncias

\begin{tabular}{lllll}
\hline muestra & mínimo & máximo & $X$ & SD \\
\hline 100 & 2,41 & 26,16 & 12,71 & 5,28 \\
\hline
\end{tabular}

Tabla 7. Comparación de tiempos de cirugía efectiva esperado y obtenido.

\begin{tabular}{lccll}
\hline muestra & tiempo obtenido & tiempo esperado & $\mathrm{x}$ & $\mathrm{p}$ \\
\hline 100 & 13,03 & 12,70 & 0,3226 & 0,748 \\
\hline
\end{tabular}

\section{Discusión}

En el presente estudio hemos podido observar que dentro de los diversos factores que influyen en el tiempo de la exodoncia del tercer molar inferior, sólo algunos parecen tener real relevancia.

La elección del tipo de radiografía y su interpretación es de suma importancia en el preoperatorio, como lo confirman Chen, Huang y Cheng (19). Según lo que se desea apreciar se elegirá la radiografía. Si bien la anatomía dentaria puede seguir un patrón establecido, también presenta variaciones individuales en el número y la forma de la raíz. Desafortunadamente, la morfología de las raíces no está siempre bien definida en las radiografías. Por ejemplo, la subestimación de la curvatura de la raíz podría conllevar a una fractura del ápice.

Bell (20) manifiesta que la radiografía panorámica es la de elección para evaluar los alcances preoperatorios del tercer molar mandibular, ya que permite observar la posición, profundidad y tipo de impactación, así como la textura del hueso. Sin embargo, señala que existen diferencias substanciales entre las características del tercer molar observadas en la radiografía y lo que se encuentra durante la operación. En radiografías panorámicas, no se puede ver en detalle las raíces de diente, por lo que White y Pharoah (21) sugieren la utilización de radiografías periapicales.

Con respecto a la ubicación, encontramos que la relación espacial más fre- cuente fue la mesioangular con un 55\%, seguido de la posición vertical con un $29 \%$. Resultados muy similares a los encontrados por Quek et al. (22), quienes encontraron un $60 \%$ de piezas ubicadas en posición mesioangular y un $10 \%$ en posición vertical. Guthua y Mwaniki (23) también encontraron que la posición más frecuente de impactación fue la mesioangular con un $72 \%$ de los casos estudiados en el hospital de Narobi, Kenya. En un estudio realizado en el Perú, Mier (18) encontró sobre un total de 151 casos un 31,13\% en la posición mesioangular y 28,48\% en la posición vertical, resultados muy similares a los encontrados por Delgado (17), quien encontró en 500 molares, $37 \%$ en posición mesionangular y $36,65 \%$ en posición vertical. Los hallazgos que se presentan en estos estudios son inversos a los que encontraron Bataineh, Albashaireh y Hazza'a (24) al estudiar la angulación del tercer molar en una población jordana donde encontraron mayor frecuencia de la posición vertical con un $61,4 \%$ seguida de la posición mesioangular con un 18,1\%. En el Perú, Deza (25) en su estudio encontró un $46,67 \%$ de las piezas dentarias estudiadas en posición vertical y 20,44\% en posición mesioangular.

La variación en los porcentajes encontrados se pueden explicar por los cambios que ocurren en la angulación de los terceros molares, durante su proceso de erupción, así lo demuestran los estudios de Venta et al. (26) y Sewerin (27).

Con relación a los niveles de profundidad, nuestro estudio encontró que el nivel A era el más frecuente con $70 \%$ de los casos, seguido por el nivel B con $25 \%$ de los casos. Este resultado es similar al encontrado por Hugoson y Kugelberg (28). Sin embargo Yuasa y Sugiura (29) hallaron que el nivel B era el más frecuente.

La edad y el grado de maduración del diente en el momento de la extracción puede influenciar en el nivel de profundidad, tal como lo explica en su estudio Venta et al. (26).

$\mathrm{Al}$ observar nuestros resultados relacionados al espacio disponible, podemos decir que obtuvimos con mayor frecuencia la clase II en un 56\% de los casos, y en frecuencias iguales a $22 \%$ aparecieron las clases I y III. Estos datos son en cierta forma similares a lo que expresa Hattab y Alhaija (30). Algunas de las causas de la insuficiencia de espacio antero posterior pueden ser explicadas por la discrepancia en el tamaño de la mandíbula debido a cambios evolutivos durante la vida o por la insuficiente elongación de la región del tercer molar.

La forma de las raíces como una de las variables, fue tomada en cuenta debido a que uno de los momentos más difíciles dentro de la cirugía del tercer molar se da en el momento de retirarlas, y esto a causa de la morfología tan variable que pueden presentar.

Sin embargo, en nuestro estudio, ninguna de las formas radiculares evaluadas fue significativa al realizar el análisis de regresión lineal. Además cuando se realizó el análisis bivariado relacionando el tiempo y la forma de la raíz, ésta no fue un factor determinante para aumentar el tiempo de cirugía efectiva y/o decir que se trataba de una extracción difícil. Situación que concuerda con lo que reportaron Yuasa, Hawai y Sugiura (3).

Después de evaluar las características radiográficas, también se evaluó dos covariables importantes: el motivo por el cuál se indica la exodoncia del tercer molar y la edad en que la cirugía se realiza.

En el presente estudio el motivo más frecuente por el cual se indicó la extrac- 
ción del tercer molar fue por prevención, de esta forma se evita la morbilidad que puede producir el tercer molar en el futuro (31).

Con respecto a la edad, en diversos estudios (12, 16, 22, 26, 29), así como en el presente, es considerada como un factor significativamente importante que afecta la cirugía del tercer molar mandibular. Así por ejemplo, Renton, Smeeton y McGurk (12) encontraron que a partir de los 30 años, las exodoncias de terceros molares eran más difíciles que aquellas que se realizaron en pacientes jóvenes, y concluyó que la dificultad era directamente proporcional a la edad. Continuando con estas afirmaciones, Yuasa y Sugiura (29) concluyen que los sucesos postoperatorios inmediatos (aumento de volumen y dolor) de una extracción de tercer molar inferior varían dependiendo de la edad del paciente, agudizándose a mayor edad.

Como se ha podido observar cada una de las variables antes mencionadas no eran fijas, es decir podían variar de un tipo a otro dentro de su clasificación dependiendo de la edad del paciente. Con lo que podemos afirmar que no es igual realizar una extracción de un tercer molar mandibular a un paciente joven con características determinadas, que realizar una exodoncia a un paciente mayor con las mismas características. Lo que a su vez, demostraría la variación en el tiempo de cirugía efectiva, ya que siguiendo la premisa anterior, tomaría más tiempo realizar una exodoncia en un paciente de mayor edad.

Entonces al relacionar todas las variables revisadas mediante un análisis bivariado de regresión lineal encontramos que sólo algunas de las variables significativas guardaban real relación con el factor tiempo. Así con respecto a las variables de ubicación, se observó que dentro de la relación espacial eran significativas las posiciones vertical, mesioangular y horizontal, pero de éstas sólo la posición vertical guarda correlación. Con respecto a la profundidad, el nivel A y B fueron significativos pero ninguno guarda correlación con el tiempo. Y con relación al espacio disponible fueron significativas las clase I y
III pero sólo guarda correlación la clase III. Cuando se evaluó la forma y número de raíces, quedamos sorprendidos al no observar una relación significativa de correlación entre éstas y el tiempo. Sucediendo la misma falta de significancia en relación al motivo por el cual se realizó la exodoncia

Por tanto, es que nuestro modelo final presenta una relación inversamente proporcional entre el tiempo y la posición vertical, es decir, se encontró que a medida que se presentaba la posición vertical menor sería el tiempo de cirugía efectiva. Mientras que se observó la existencia de una relación directamente proporcional entre tiempo - clase III y tiempo edad, es decir que a medida que se presentaba la clase III y a mayor edad del paciente, el tiempo seria también mucho mayor. Lo que concuerda en cierta forma con los modelos de Hattab y Alhaija (30), Yuasa, Hawai y Sugiura (3).

No existe mucha bibliografía con respecto al tiempo de cirugía efectiva, pero sí con relación al tiempo operatorio y a factores que facilitan o dificultan la extracción. Es por eso que se ha querido evaluar el tiempo de cirugía efectiva de la exodoncia del tercer molar, y los factores que influyen sobre éste.

Finalmente, consideramos que la experiencia del operador es un factor que influye en los resultados obtenidos, de lo que se puede concluir que a más años de experiencia en la especialidad es menor el tiempo de cirugía efectiva. Y es por eso que se han observado que sólo tres de las variables estudiadas entran en el modelo final del análisis multivariado.

Así mismo, consideramos que deben realizarse estudios similares donde el operador tenga menos años de experiencia, ya que si bien en este caso no hemos encontrado influencia de las raíces, creemos que esto se debe a la experiencia del operador.

Si se presentaran situaciones similares en operadores de menor experiencia probablemente no sólo serían tres las variables que presentarían relación con el tiempo. Existirían otras, como por ejemplo la forma de las raíces, las que podrían variar el tiempo de la cirugía efectiva del tercer molar mandibular.

\section{Referencias bibliográficas}

1. Gay Escoda C, Berini Aytés L. Cirugía bucal. Madrid: Ergón SA; 1999.

2. Kouame P, Souaga K, Amantchi D. Difficulties in the surgery of lower wisdom teeth in dental offices in Ivory Coast. Odontostomatol Trop 1998; 21(84):15-7.

3. Yuasa H, Kawai T, Sugiura M. Classification of surgical difficulty in extracting impacted third molars. Br J Oral Maxillofac Surg 2002; 40(1):26-31.

4. Berge TI, Gilhuus-Moe OT. Per- and post-operative variables of mandibular third-molar surgery by four general practitioners and one oral surgeon. Acta Odontol Scand 1993; 51(6):389-97.

5. Pifarré Sahuja E. Patología quirúrgica oral y maxilofacial. Barcelona: Editorial JIMS; 1993.

6. Seminario A. Frecuencia de las complicaciones post exodoncia de terceros molares mandibulares en el servicio de Medicina, Cirugía y Patología Oral [Tesis]. Lima: Universidad Cayetano Heredia; 1997.

7. Laskin D. Cirugía bucal y maxilofacial. Buenos Aires: Editorial Medica Panamericana; 1987.

8. Ramadas Y, Sealey CM. Third molar removal and nerve injury. N Z Dent J 2001; 97(427):25-8.

9. Heurich T, Ziegler C, Steveling $\mathrm{H}$, Wortche R, Muhling J, Hassfeld S. Digital volume tomography--an extension to the diagnostic procedures available for application before surgical removal of third molars. Mund Kiefer Gesichtschir 2002; 6(6):427-32.

10. Loescher AR, Smith KG, Robinson PP. Nerve damage and third molar removal. Dent Update 2003; 30(7):375-82.

11. Bell GW, Rodgers JM, Grime RJ, Edwards KL, Hahn MR, Dorman ML, et al. The accuracy of dental panoramic tomographs in determining the root morphology of mandibular third molar teeth before surgery. Oral Surg Oral Med Oral Pathol Oral Radiol Endod 2003; 95(1):119-25.

12. Renton T, Smeeton N, McGurk M. 
Factors predictive of difficulty of mandibular third molar surgery. $\mathrm{Br}$ Dent J 2001; 190(11):607-10.

13. Garcia AG, Sampedro FG, Rey JG, Vila PG, Martin MS. Pell-Gregory classification is unreliable as a predictor of difficulty in extracting impacted lower third molars. Br J Oral Maxillofac Surg 2000; 38(6):585-587.

14. Koerner K. Métodos sin misterio. Exodoncias de terceros molares mandibulares. Dent Today 1994/ 1995; 6(3):19-33.

15. Archer WH. Oral and maxillofacial surgery. 5 ed. Philadelphia: WB Saunders; 1979.

16. Reyneke JP, Tsakiris P, Becker P. Age as a factor in the complication rate after removal of unerupted/impacted third molars at the time of mandibular sagital split osteotomy. J Oral Maxillofac Surg 2002; 60(6):654-9.

17. Delgado I. Características de los terceros molares inferiores impactados observados en las radiografías panorámicas de pacientes atendidos en la Clínica Estomatológica Central de la Facultad de Estomatología, UPCH [Tesis]. Lima: Universidad Cayetano Heredia; 2001.

18. Mier G. Comparación entre el índice de dificultad con el tiempo de cirugía efectiva en la exodoncia del tercer molar inferior realizada por residentes en el servicio de cirugía oral y maxilofacial de la Universidad Peruana Cayetano Heredia en el periodo Febrero-Marzo 2002 [Tesis]. Lima:
Universidad Cayetano Heredia; 2002.

19. Chen SK, Huang GF, Cheng SJ. The relationship between radiologic interpretation and root tip fracture during tooth extraction performed by junior clinicians. Oral Surg Oral Med Oral Pathol Oral Radiol Endod 2001; 92(4):470-2.

20. Bell GW. Use of dental panoramic tomographs to predict the relation between mandibular third molar teeth and the inferior alveolar nerve. Radiological and surgical findings, and clinical outcome. Br J Oral Maxillofac Surg 2004; 42(1):21-7.

21. White SC, Pharoah MJ. Oral radiology. Principles and interpretation. 4 ed St. Louis: Mosby Co; 2000.

22. Quek SL, Tay CK, Tay KH, Toh SL, Lim KC. Pattern of third molar impaction in a Singapore Chinese population: a retrospective radiographic survey. Int J Oral Maxillofac Surg 2003; 32(5):548-52.

23. Guthua SW, Mwaniki DL. A retrospective study of characteristics of impacted mandibular wisdom teeth in 110 patients treated in Nairobi, Kenya. Afr Dent J 1992; 6:30-3.

24. Bataineh AB, Albashaireh ZS, Hazza'a AM. The surgical removal of mandibular third molars: a study in decision making. Quintessence Int 2002; 33(8):613-7.

25. Deza G. Frecuencia de las posiciones angulares más comunes de los terce- ros molares mandibulares de los pacientes que acudieron a la clínica Estomatológica Central de la Universidad Peruana Cayetano Heredia durante el año 1998 [Tesis]. Lima: Universidad Cayetano Heredia; 1999.

26. Venta I, Turtola L, Ylipaavalniemi P. Radiographic follow-up of impacted third molars from age 20 to 32 years. Int J Oral Maxillofac Surg 2001; 30(1):54-7.

27. Sewerin I, von Wowern N. A radiographic four-year follow-up study of asymptomatic mandibular third molars in young adults. Int Dent J 1990; 40(1):24-30.

28. Hugoson A, Kugelberg CF. The prevalence of third molars in a Swedish population. An epidemiological study. Community Dent Health 1988; 5(2):121-38.

29. Yuasa H, Sugiura M. Clinical postoperative findings after removal of impacted mandibular third molars: prediction of postoperative facial swelling and pain based on preoperative variables. Br J Oral Maxillofac Surg 2004; 42(3):209-14.

30. Hattab FN, Alhaija ES. Radiographic evaluation of mandibular third molar eruption space. Oral Surg Oral Med Oral Pathol Oral Radiol Endod 1999; 88(3):285-91.

31. Silvestri AR Jr, Singh I. The unresolved problem of the third molar: would people be better off without it? J Am Dent Assoc 2003; 134(4):450-5. 